\title{
Describing categories of objects for menu retrieval systems
}

\author{
SUSAN T. DUMAIS and THOMAS K. LANDAUER \\ Bell Communications Research, Inc., Murray Hill, New Jersey
}

\begin{abstract}
When searching for information in menu-based retrieval systems, users are given a choice among a set of categories. Successful retrieval of information depends critically on the user's understanding the system's division of objects into categories and the system's labels for these categories. This research compares several different ways of describing ill-defined categories of objects using combinations of names and examples. Examples provide a promising possibility, both as a means of flexibly naming new or difficult menu categories and as a methodological tool for studying certain categorization problems.
\end{abstract}

This paper describes some recent work on describing ill-defined categories of objects and the implications this has for information retrieval. Menu access forms the basis of many supposedly "user-friendly" information retrieval and database access systems. In menu systems, users are presented with a list of category descriptions and must choose the category to which items of interest belong. The choices are typically arranged in a hierarchy of menus such that choices at one level lead to a refinement of the selected category. A concrete example may help to illustrate the problem and the types of domains and categories in which we were interested. Figure 1A shows a top-level menu page based roughly on the British Prestel videotex system. A user interested in obtaining information about local restaurants would probably select option number 3 (although options 6 and 10 are also possibilities). Figure $1 \mathrm{~B}$ shows the refinement of the entertainment category that the user would see. Selection proceeds in this manner until the desired information is located. As can be seen, such systems contain a wide variety of information that is described to users by means of labeled categories that vary in their descriptive adequacy.

Menu-based retrieval systems like this are widely used. Among the purported advantages of menu systems is that they structure the user's task in several ways (Allen, 1983). First, lists of currently available options are always displayed, so even naive users are not at a complete loss for what to do. Further, tree organizations provide an efficient and sufficient way to traverse the database and a possible way to convey important relationships among categories of objects (e.g., superordination, subordination). However, menu systems are not without problems. They can be tedious for experi-

The work reported in this paper was done while the authors were at AT\&T Bell Laboratories. The authors' mailing address is: Bell Communications Research, Inc., 600 Mountain Avenue, Murray Hill, NJ 07947.
(A) Top-Level Menu

1. News and Weather

2. Sports and Hobbies

3. Entertainment

4. Holidays, Transport, Travel

5. Marketplace

6. Advice

7. Government Information

8. Employment

9. Books and Reference

10. Other Information

(B) Menu Seen as a Result of Selecting Option No. 3 Above

1. Games You Can Play on Prestel

2. What's On

3. Music

4. Places and Buildings to Visit

5. Stories

6. Sports and Hobbies

7. Restaurants and Pubs

8. Fun Page

9. Horoscopes

Figure 1. Example of menus of options that might be found in existing home information or videotex systems. Panel A shows a top-level menu, and Panel $B$ shows the refinement of the entertainment category (option number 3 ).

enced users; not all kinds of information (Brosey \& Shneiderman, 1978; Durding, Becker, \& Gould, 1977) or tasks (Landauer, Dumais, Gomez, \& Furnas, 1982) are equally suitable for hierarchical presentation; and the search and access routes depend on a rigid, partly arbitrary organization imposed by system designers. The last point is particularly important. Menus are a categorization of information. The contents of a system are described in terms of sets of categories, and users must be able to predict the contents of these categories in order to find information. The success of a menu organization depends on effectively grouping large sets of objects and on describing the resulting categories so that people can anticipate their contents. 
At first glance, one might think that knowing what is in such categories is not much of a problem. After all, our ability to divide the world into categories is an important and pervasive aspect of human thought. However, natural categories lack many properties desirable in information retrieval contexts. They are seldom compact, distinct, regularly organized, precisely defined, or easy to describe. Instead, natural categories are "fuzzy," both in the sense that they overlap with each other and in the sense that many items are only probabilistically contained in them (Rosch, 1978); they are related by complex and not necessarily hierarchical relations that can vary over people, time, and tasks; they can be defined in several ways (enumeration, shared attributes, family resemblance, similarity to an archetype, etc.), some more precise than others; and they are not always easy to describe precisely or concisely. These are serious problems for menu-based infor. mation retrieval, and deserve elaboration.

Figure 2 presents what we think are two important classes of psychological problems in categorizing information for menu access. Young and Hull $(1982,1983)$ have also reviewed several kinds of problems that users encounter in selecting among menu options. The "category naming" problem is of particular interest in this paper. There are several components of the naming problem. At a fairly superficial level, category names are not always highly descriptive or informative. Category names are sometimes inadvertently (or intentionally) vague. Menu options like "Other Information," "Miscellaneous," and "Generalities" are often difficult for users to interpret. For example, one probably would not have guessed that computer science books are categorized under the heading "Generalities" in the Dewey Decimal System. Other times, category names are intentionally attractive or cute, but not necessarily communicative, especially for new users. Although the category names "What's On" and "Fun Page" are catchy, they may not clearly convey their contents. Finally, some categories are just hard to describe with a short name. Even when

\section{Inaccurate Category Names \\ a. category names not always descriptive - vague (e.g., "other"; "generalities"; “miscellaneous") - cute (e.g., "what's on"; "fun page") - hard to name categories \\ b. mismatch between system designer's and user's interpretation of the category name \\ 2. Classification \\ a. overlapping and fuzzy categories \\ b. classification on the basis of one (or a few) dimensions or aspects}

Figure 2. Two of the major classes of problems in the design of menu-based information retrieval situations. The first concerns the difficulty in naming categories of objects so that users can understand their contents. The second problem is concerned with the difficulties involved in partitioning large sets of diverse objects. the name is "understood," there can be a mismatch between the designer's understanding of a category name and the user's or between the items actually available and the set of items implied by the title. Ideally, a category title should be taken by the user to refer to all and only those things available in the system under that description. Category names seldom meet this criterion. Instead, short category names or descriptive phrases will almost necessarily lead users to a too narrow, a too broad, or simply an inaccurate interpretation of the actual set of items stored as a given referenced category.

The second class of problems has to do with classification. Although these problems are important, they are not the primary focus of this paper and are discussed only briefly. Real categories are often overlapping and fuzzy, making unambiguous partitioning impossible. Furthermore, objects in menu systems are usually classified according to one (or at most a few) aspects or dimensions. There is no guarantee that the aspects implicit in the system categorization are the ones by which a particular user needs to access the information in a particular situation, or that any single aspect will be useful in many different situations.

Many systems of interest, such as videotex or home information systems, consist of new and frequently changing content in large and varied domains, and are particularly prone to the problems described above. Experimental evaluations of existing videotex systems (e.g., Prestel, Viditel, Antiope, and Telidon) have, in fact, shown that people have great difficulty in finding representative items (Frankhuizen \& Vrins, 1980; McEwen, 1981; Vrins, van Velthoven, \& Frankhuizen, 1982; Whalen \& Latremouille, 1981). ${ }^{1}$ (See also Dillon $\&$ Tombaugh, 1982, for a review of some of the more general psychological research and issues in videotex systems.) In the Frankhuizen and Vrins (1980) study, for example, a version of the Prestel viewdata service was examined. This system contains a wide variety of information about such diverse topics as news, weather, sports, travel, financial, and government information, and reference materials. Twelve subjects were given questions such as "What language is spoken in Ethiopia?" or "What can you do when your child swallows poison?" and were asked to find relevant information in the database. The main result was that information could not easily be found. More specifically, only $56 \%$ of the questions were answered correctly; in $46 \%$ of the problems, subjects started over from the beginning at least once; and even when they were successful in finding relevant information, users accessed an average of twice as many pages as were strictly necessary. The authors reported that many of the problems novice users encountered with the system were essentially those mentioned above: category overlap and category naming. Not surprisingly, subjects also experienced difficulties when the information they were look. 
ing for was not in the database. Although this is likely to be a common and important problem in real-world situations, research in this area is just beginning (Whalen \& Latremouille, 1981).

We have been looking at one way in which category descriptions might be improved. In many commercially available systems, categories are often described by means of short names or labels. Interestingly, much of our knowledge about natural categories comes from experience with examples of the category, as opposed to more formal descriptions, rules, or definitions. Research in concept learning and schema abstraction has shown that examples are important both in category formation and in subsequent categorization of new instances (Smith \& Medin, 1981). It seems, then, that examples could be useful in describing new and varied categories of objects in menu retrieval contexts; and, indeed, they are often used in a supplementary style in practice. ${ }^{2}$ Examples have the additional presumptive virtue of embellishing and making more concrete abstract category descriptions.

\section{EXPERIMENTS}

We have begun some work aimed at better understanding how examples can be used in the description of categories of objects. We examined categorization in a relatively difficult domain-Yellow Page headings (i.e., items such as "lawyers," "moving and storage," and "party supplies"). This domain has many interesting charcteristics similar to those found in home information systems. It covers a wide range of objects and services, and it is not easily partitioned into a few large and mutually exclusive superordinate categories. In part, this is because no conventional categories exist at high levels of superordination for these items.

\section{Method}

Obtaining categories. We first created categories of Yellow Page headings. A typical phone book contains 2,000-3,000 headings. We selected a manageable subset (307) of the headings with the largest number of entries in the Suburban Essex, New Jersey Bell Yellow Pages. We then applied systematic scaling techniques to generate superordinate categories. It is important to note that these were not intended to be psychologically optimal categories, although we do know more about their perceived structure than is often the case in real systems. Their advantage is that they were partitions of the desired size and level of $a b$ straction and provided a vehicle to study the assignment and use of various category naming schemes.

Three hundred and ten New York University undergraduates sorted subsets of the Yellow Page headings into categories. Each student was given a sheet of paper containing a balanced subset of 153 of the headings and was asked to group them into five superordinate categories, each with 15 or more items. They did this by placing a number 1 to 5 next to the items that belonged together. They were free to group items in any way they wished and were not required to categorize all items.

For each pair of items, we calculated the proportion of times that the pair was placed in the same category. This generated a $(307 \times 306) / 2$ half-matrix of similarities, which was used as input to a hierarchical clustering program based on Johnson's (1967) scheme. Five large (greater than 35 headings) and relatively good (joining as near the top of the tree as possible, given the size constraints) clusters were selected from the resulting median method. These superordinate categories are groupings of Yellow Page listings, like those that might be encountered at an early level of menu access. Figure 3 shows the items in the five resulting categories. Like many natural categories, these clearly lack many desirable properties for information retrieval, such as familiarity, concreteness, compactness, discriminability, and ease of description. It is intuitively clear that this was not a very good method of categorization. But, it did yield a reasonable set of representatively bad categories with which to explore category naming issues (and it appears, from aspects of our results not reported here, that it may not be easy to do better).

Describing categories. We wanted to see if useful names could be found for such new, ill-defined, and high-level categories. We studied several different ways of communicating the contents of these categories to people. First, we asked 15 "experts" to assign descriptive labels or phrases to each of the categories. This is typically done in commercial systems, in which either domain or classification experts are used. Our experts included library scientists, linguists, computer scientists, psychometricians, and cognitive psychologists. We gave each expert lists of all the headings in each category and had them assign a name or short descriptive phrase to each category. (Incidentally, they found this task quite difficult.) In addition, we tried describing the contents of each category by giving "examples" of items in the category. Three methods of choosing examples were examined: (1) random selection; (2) selection by human experts; and (3) selection by a computer algorithm that essentially spread examples uniformly over the category. ${ }^{3}$

Thus, there were several different ways of describing our categories of headings, depending on: (1) how the categories were described (names alone, examples alone, or names and examples); (2) how the examples were chosen (randomly, by experts, or by algorithm); and (3) how many examples were used (1 or 3 ).

We next evaluated people's ability to choose among a menu of categories described in these different ways. We gave over 700 SUNY-Stony Brook students 72 randomly chosen target items from our original sample of headings and had them decide to which category each item belonged. There were 10 headings from each of the five categories and 22 headings from the same original sample that did not, according to the clustering solution, belong to any of the five categories. (Twelve of these 22 items were used as part of an auxiliary demonstration and were not scored in any of the analyses reported below.) Subjects were given a sheet of paper with the five categories "named" in one of the ways described above, plus a miscellaneous category simply labeled "miscellaneous." In the case of expert names or examples, any given subject was shown the full set of category names, or examples, offered by one of the 15 experts, the particular expert being varied over subjects. The 72 target headings were numbered 1 to 72 and were sequentially presented for about $10 \mathrm{sec}$ each. Subjects indicated to which category they thought the target belonged by placing the corresponding number under the appropriate category description. If they did not think a target heading belonged in one of the five named categories, they were instructed to put it in the miscellaneous category. Subjects received no feedback about their decisions.

\section{Results}

The main results of interest are shown in Figure 4. The numbers presented are the proportions of items correctly categorized into one of the five named categories; standard errors about subject means are shown in 
Categories Obtained by Hierarchical Clustering

\begin{tabular}{|c|c|c|c|c|}
\hline Category 1 & Category 2 & Category 3 & Category 4 & Category 5 \\
\hline tire dealers & chemicals & bathroom remodeling & microfilming & television \& radio \\
\hline translators & scaffolding & delivery service & associations & home improvements \\
\hline opticians & dictating machines & brake service & fabric shops & ceramics \\
\hline stock brokers & machine shops & garbage collection & real estate & sewing machines \\
\hline interior decorators & hardware & waterproofing & insurance & vacuum cleaners \\
\hline engineers & automobiles & building cleaning & apartments & pets \\
\hline locks \& locksmiths & cash registers & detective agencies & clinics & kitchen cabinets \\
\hline tailors & elevators & employment agencies & bridal shops & furniture \\
\hline dressmakers & sand \& gravel & typing service & labor organizations & lamps \\
\hline plumbers & garden $\&$ lawn eqpt. & towing & mailing lists & nurseries-plants \\
\hline cleaners & motorcycles & weight control & day nurseries & awnings \& canopies \\
\hline marriage counselors & welding & recording service & signs & water heaters \\
\hline dentists & buses & house cleaning & hospitals & windows \\
\hline surveyors & scrap metals & sodding service & loans & railings \\
\hline architects & wheel chairs & floor refinishing & churches & tropical fish \\
\hline jewelers & audio-visual eqpt. & ambulances & typesetting & paint \\
\hline excavators & pumps & collection agencies & stables & stereo eqpt. \\
\hline adjusters & packaging materials & wheel alignment & mortgages & venetian blinds \\
\hline heating contractors & hearing aids & tree service & word processing & dishwashers \\
\hline nurses & trailers & hair replacement & financing & wallpapers \\
\hline paving contractors & engines & rental service & business forms & screens \\
\hline security guards & burglar alarms & exterminating & gift shops & juvenile furniture \\
\hline roofers & snow removal eqpt. & car washing & pension plans & camping eqpt. \\
\hline barbers & trucks & janitor service & beauty salons & draperies \\
\hline entertainers & sprinklers & water companies & cemeteries & oil burners \\
\hline clergy & laboratory eqpt. & chimney cleaning & beauty schools & party supplies \\
\hline caterers & monuments & driving instruction & nursing homes & lighting fixtures \\
\hline auctioneers & cranes & messenger service & schools & lawn mowers \\
\hline siding contractors & scales & electrolysis & kennels & video recorders \\
\hline contractors & garbage disposals & answering service & language schools & washing machines \\
\hline upholsterers & warehouses & resume service & family planning & artists' supplies \\
\hline painters & tools-electric & limousine service & screen printing & water softening \\
\hline lawyers & sheet metal work & taxicabs & data processing & carpets \& rugs \\
\hline landscapers & industrial eqpt. & window cleaning & vocational guidance & plants \\
\hline printers & vending machines & moving \& storage & banks & refrigerators \\
\hline social workets & photographic eqpt. & advertising & synagogues & gutters \\
\hline physicians & air compressors & & pharmacies & billiard eqpt. \\
\hline coin dealers & copying machines & & social services & stoves \\
\hline book dealers & tractors & & department stores & newspapers \\
\hline psychologists & riggers & & fund raising & mattresses \\
\hline optometrists & typewriters & & laundries & ranges \& ovens \\
\hline veterinarians & computers & & alcoholism info. & fences \\
\hline electricians & mufflers & & & glass \\
\hline boat dealers & topsoil & & & appliances \\
\hline stationers & fans & & & \\
\hline florists & solar energy eqpt. & & & \\
\hline steel fabricators & time recorders & & & \\
\hline chiropractors & safes \& vaults & & & \\
\hline bakers & fire extinguishers & & & \\
\hline physical therapists & air cargo & & & \\
\hline funeral directors & fireplace eqpt. & & & \\
\hline cabinet nakers & telephone eqpt. & & & \\
\hline grocers & transmissions & & & \\
\hline photographers & generators & & & \\
\hline builders & air conditioners & & & \\
\hline accountants & adding machines & & & \\
\hline
\end{tabular}

Figure 3. Items found in the five superordinate categories obtained by hierarchical clustering of Yellow Page headings. As can be seen, these categories lack many properties desirable in information retrieval contexts, such as: familiarity, concreteness, and ease of description. 


\begin{tabular}{lllll} 
& \multicolumn{2}{c}{ Name + Example(s) } & \multicolumn{2}{c}{ Just Examples } \\
\hline \multirow{2}{*}{0 Examples } & mean & $.46(.02)$ & chance $\sim .17$ \\
& random & $.49(.01)$ & random & $.23(.02)$ \\
1 Example & expert & $.47(.02)$ & expert & $.29(.02)$ \\
& algor. & $.51(.02)$ & algor. & $.30(.03)$ \\
\cline { 2 - 5 } & mean & .49 & mean & .27 \\
& random & $.54(.01)$ & random & $.45(.02)$ \\
& expert & $.51(.02)$ & expert & $.48(.02)$ \\
& algor. & $.51(.02)$ & algor. & $.50(.02)$ \\
& mean & .52 & mean & .48 \\
\hline
\end{tabular}

Figure 4. Data from the 13 main conditions of the categorization study. These numbers represent the proportion of items correctly categorized into one of the five named categories. Standard errors about subject means are shown in parentheses.

parentheses. ${ }^{4}$ First, note that overall performance was not very good. On the average, subjects correctly categorized target headings less than $50 \%$ of the time (with a range of $23 \%$ to $54 \%$, depending on condition). Of course, this was not entirely unexpected, since we intentionally chose a very difficult domain. Furthermore, as mentioned above, although our classification procedure was systematic, it was not necessarily a psychologically optimal one.

A striking aspect of these data is the poor performance with categories described by just a single example (.27)-note that chance is approximately .17 . This accuracy is reliably different from the accuracy levels observed in the other four major naming conditions, according to Newman-Keuls paired comparisons at the .01 level. [The overall analysis of variance for the five major conditions shown in Figure 4 is highly reliable: $F(4,701)$ $=82.02, \mathrm{p}<.0001$.] On closer examination of the data from this condition, we found that subjects put $41 \%$ of the target items in the miscellaneous category (as compared with an average of $20 \%$ for the other naming conditions). Apparently, if subjects could not easily place a target in one of the five specific categories, they put it in "miscellaneous." The observation that subjects put many items in miscellaneous raises the possibility that part of the poor performance is a result of an overly strict criterion, rather than a true lack of discriminability among the categories.

To explore this possibility, we gathered some additional data for a subset of the original conditions, one or three algorithmically chosen examples, with the miscellaneous category removed from the set of menu alternatives. Subjects were an additional 49 New York University undergraduates. The procedure was exactly the same as in the previous study, except that the miscellaneous option was not included in the menu of choice alternatives. Performance improved from the previous .30 to .44 in the one-example condition and from .5C to .61 in the three-example condition. Corrected for guessing (because there are fewer choices and a higher chance of guessing correctly in the no-miscellaneous experiment), performance improved by over $100 \%$ in the one-example naming condition (.16 to .33$)$ and $30 \%$ in the three-example condition (.40 to .52$)$. This suggests that people use excessive caution in the presence of the ill-defined miscellaneous category. Another possibility, however, is that a vague category name, like miscellaneous, degrades subjects' understanding and use of the other category choices. This interpretation is consistent with work by Whalen and Mason (1981) on the Telidon videotex system; Whalen and Mason found that the presence of vague category labels impaired performance. $^{5}$

The most interesting and encouraging aspect of our results concerns the use of examples. We found that three examples alone (.48) were essentially as good a way of describing these categories as were expert-chosen category names alone (.46) or as names plus examples (.50 average). Note that even randomly chosen examples (.45) were about as good as expertly chosen names. (None of these comparisons are reliable according to Newman-Keuls tests at the .05 level.) This type of exemplification offers the possibility of relatively automatic and flexible ways of naming categories. When names are unavailable or when it is desirable to categorize a large set of items in ad hoc ways, examples may offer an attractive way to "name" the categories. This result is also nice from a methodological point of view. Many interesting experiments about alternative categorization schemes (e.g., depth-breadth tradeoffs, or optimal number of category alternatives) involve partitioning a set of objects in several different ways. One problem that plagues this research is equating the goodness or appropriateness of category names across different partitions of the information. The use of examples provides a possible means for overcoming some aspects of this problem.

One final aspect of the data concerns the effectiveness of examples chosen in different ways. A $2 \times 2 \times 3$ analysis of the three perfectly crossed factors (how categories described-name plus example vs. just examples; number of examples -1 vs. 3 ; and how examples chosen-random, expert, algorithm) shows a marginally significant effect of how the examples were selected: $.42, .44, .46$, for the randomly, expertly, and algorithmically chosen examples, respectively $[\mathrm{F}(2,640)=2.82$, $\mathrm{p}=.06]$. An interpretation of this effect is qualified by a reliable interaction between how examples were chosen and how the categories were described $[F(2,640)$ $=4.52, p=.01]$. Essentially, the way in which examples were chosen had little or no systematic effect if a name was used in the description (.51 random, .49 expert, .51 algorithm); but, having carefully chosen examples was important when examples alone were used to describe the categories (.33 random, .39 expert, .40 algorithm). One interpretation of this result is that information about the central tendency of categories 
is required for effective categorization. Category names provide this information, and apparently so do carefully chosen examples. ${ }^{6}$ Although randomly chosen examples result in somewhat inferior categorization accuracy when they provide the only means of category description, it is possible that adding an additional randomly chosen example would improve performance to the level observed for carefully chosen examples. This would be especially useful in practical situations, in which experts or interitem similarity measures are not always available. Finally, the good performance observed in the "name plus three random examples" condition raises an intriguing possibility. Perhaps the best way to convey the contents of a category is to provide information about both central tendency and variability. The name provides central tendency information, and the randomly chosen examples may have added information about the range of objects included in the categories. (The algorithmic examples were chosen explicitly to minimize the summed distance from other items in the set, so they provide little direct information about the extremes of the category; and, expertly chosen examples may also have been more central members.)

\section{SUMMARY}

Several conclusions can be drawn from the categorization study. Overall accuracy was only about 50\%, suggesting that automatic construction of good categories in difficult domains is not an easy problem. Performance in the one-example naming condition was quite low, but this was due, in part at least, to an excessively high criterion for classifying items when a vague category descriptor, like miscellaneous, was present. The most interesting and important result was that three examples were as effective a way of describing our categories as either expertly chosen names or names plus examples. This provides a relatively automatic and flexible way of describing categories of objects for information retrieval. The way in which examples are selected is most important when they provide the only means of category description. The technique of describing categories by means of example can also be used to explore several issues in menu design.

As noted above, the work reported in this paper focused on the category naming problem. Certainly, more work needs to be done in this area, and we are currently exploring several quantitative aspects of the menu selection process. Other important research directions are more directly concerned with classification issues-that is, how to effectively divide large domains into useful categories. Although most current menu systems are hierarchical trees with partitions at each node, more complicated graph structures and relations should be fruitful areas for future explorations.

\section{REFERENCES}

Allen, R. B. (1983). Cognitive factors in the use of menus and trees: An experiment. IEEE Journal on Selected Areas in Communications, 1, 333-336.

Brosey, M., \& Shneiderman, B. (1978). Two experimental comparisons of relational and hierarchical data models. International Journal of Man-Machine Studies, 10, 625-637.

Dillon, R. F., \& Tombaugh, J. W. (1982). Psychological research on videotex. Behavior Research Methods \& Instrumentation, 14, 191-197.

Dumais, S. T., \& Landauer, T. K. (1983). Using examples to describe categories (Internal memorandum). Murray Hill, NJ: Bell Laboratories.

Durding, B. M., Becker, C. A., \& Gould, J. D. (1977). Data organization. Human Factors, 19, 1-14.

Frankhuizen, J. L., \& VRins, N. (1980). Human factors studies with Viewdata. In Proceedings of the Ninth International Symposium on Human Factors in Telecommunications (pp. 1-7).

Johnson, S. C. (1967). Hierarchical clustering schemes. Psychometrika, 32, 241-254.

KIGER, J. L. (1984). The depth/breadth tradeoff in the design of menu driven user interfaces. International Journal of ManMachine Studies, 20, 201-213.

Landauer, T. K., Dumais, S. T., Gomez, L. M., \& Furnas, G. W. (1982). Human factors in data access. Bell System Technical Journal, 61, 2487-2509.

McEWEn, S. A. (1981, May). An investigation of user search performance on a Telidon information retrieval system (Telidon Behavioral Research 2: The Design of Videotex Tree Indices, pp. 35-61). Ottawa, Canada: Department of Communications.

MilLER, D. P. (1981). The depth/breadth tradeoff in hierarchical computer menus. In R. C. Sugarman (Ed.), Proceedings of the Human Factors Society (pp. 296-300). Santa Monica, CA: Human Factors Society.

Rosch, E. (1978). Principles of categorization. In E. Rosch \& B. B. Lloyd (Eds.), Cognition and categorization (pp. 27-48). Hillsdale, NJ: Erlbaum.

Smith, E. E., \& Medin, D. L. (1981). Concepts and categories, Cambridge, MA: Harvard University Press.

Somberg, B. L., \& Picardi, M. C. (1983). Locus of the information familiarity effect in the search of computer menus. In A. T. Pope \& L. D. Haugh (Eds.), Proceedings of the Human Factors Society (pp. 826-830). Santa Monica, CA: Human Factors Society.

Tou, F. N., Williams, M. D., Fikes, R., Henderson, A., \& MALONE, T. (1981). RABBIT: An intelligent database assistant. In Proceedings of the National Conference on Artificial Intelligence (pp. 314-318). Los Altos, CA: Kaufmann.

Vrins, A. G. M., VAN Velthoven, R. H., \& Frankhuizen, J. L. (1982, April). Search method evaluation in the Dutch videotex system. Displays, pp. 101-105.

Whalen, T., \& Latremouille, S. (1981, May). The effectiveness of a tree-structured index when the existence of information is uncertain (Telidon Behavioral Research 2: The Design of Videotex Tree Indices, pp. 3-12). Ottawa, Canada: Department of Communications.

Whalen, T., \& Mason, C. (1981, May). The use of tree-structured index which contains three types of design defects (Telidon Behavioral Research 2: The Design of Videotex Tree Indices, pp. 15-32). Ottawa, Canada: Department of Communications.

Young, R. M., \& Hull, A. (1982). Cognitive aspects of the selection of viewdata options by casual users. In M. B. Williams (Ed.), Proceedings of the 6th International Conference on Computer Communications (pp. 571-576). New York: NorthHolland. 
Young, R. M., \& Hull, A. (1983). Categorisation structures in hierarchical menus. In Proceedings of the Tenth International Symposium on Human Factors in Telecommunications.

\section{NOTES}

1. In other studies of performance in menu systems, success rates of $95 \%$ or better on each menu have been observed (Allen, 1983; Kiger, 1984; Miller, 1981; Somberg \& Picardi, 1983). However, the object domains used in these studies were all small and carefully chosen, fell into familiar and highly discriminable categories, and were usually well-practiced. These authors were interested in other issues, such as depth-breadth tradeoffs in the design of tree-structured menus (Kiger, 1984, and Miller, 1981) and quantitative analysis of some cognitive factors in the use of menus and trees (Allen, 1982, and Somberg \& Picardi, 1983). It is worth noting that some of the conclusions reached in those studies may be of limited generality because of the special selection of categories. In particular, studies of depth and breadth effects may be seriously confounded by the varying adequacy of category labels for different subdivisions, some of which may correspond better than others to known category names.

2. Tou, Williams, Fikes, Henderson, and Malone (1982) recently proposed an information retrieval system (RABBIT) that relies heavily on the use of examples. In their system, query formulation is an iterative process in which examples are* used to aid users in articulating tacit knowledge and in reformulating queries.
3. More precisely, the algorithm selected the one or three examples in each category that minimized the summed distance of all items to their nearest neighbor in the example set. This set of examples has high family resemblance to the other members of the category.

4. For these analyses, an item was counted as correctly categorized if it was placed in the category from which it had been selected, as defined by the clustering solution. One can redefine the category to which an item belongs by using the data from this categorization study-that is, data about where most subjects put an item can be used to define the "correct" category. If we do this, there is, of course, an increase in accuracy, but it is small in magnitude and does not interact with any variables of interest.

5. In addition, we have collected reaction time data and have found interesting changes in the menu selection process, from a self-terminating evaluation of the alternatives when the miscellaneous category was present to an exhaustive one when it was omitted (Dumais \& Landauer, 1983). These results suggest that the presence of vague categories influences people's understanding of or strategies for processing the other category choices.

6. Abstraction of central tendency information from examples could occur in several ways: by explicit generation of a category name or prototypical example, or by evaluation of relations among the examples or between new instances and the examples. In any event, whatever information is used for categorization of new instances, it is best conveyed by means of expertly chosen examples or by examples explicitly chosen to span the category. 